\title{
Adverse Drug Reactions in Management of Multi Drug Resistant Tuberculosis, in Tertiary Chest Institute
}

\author{
J. S. Akshata1, Anushree Chakrabarthy'1, R. Swapna1, Shashidhar Buggi², M. Somashekar1 \\ ${ }^{1}$ Deptartment of Pulmonary Medicine, SDS TB Research Centre and RGICD, Bangalore, India \\ ${ }^{2}$ Department of CTVS, SDS TB Research Centre and RGICD, Bangalore, India \\ Email: drjsakshata@gmail.com
}

Received 28 February 2015; accepted 9 April 2015; published 16 April 2015

Copyright (C) 2015 by authors and Scientific Research Publishing Inc.

This work is licensed under the Creative Commons Attribution International License (CC BY). http://creativecommons.org/licenses/by/4.0/

cC) (i) Open Access

\begin{abstract}
Background: Multidrug resistant tuberculosis is a global threat. Effective treatment is implemented as per RNTCP guidelines. But the drugs used have great potential to develop adverse drug reactions. Such drug reactions if not managed optimally can lead to unfavourable treatment outcome. Hence, the study is to know the occurrence of adverse drug reactions. Aims: To study the occurrence of adverse drug reactions in treatment of multidrug resistant tuberculosis and hence the factors affecting the treatment. Settings and Design: Retrospective analysis of patients treated with standardised regimen for MDR-TB, as per RNTCP guidelines at a tertiary chest institute between august 2011 and December 2014. Methods and Material: Retrospective analysis of 607 patients' records reviewed for the occurrence of adverse drug reactions. All adverse reactions are noted and diagnosed either clinically or by laboratory evidence. Results: Among the 607 patients included in the study, majority had one or more adverse drug reactions. The most common was gastritis $(71.7 \%)$, which was easily treatable, and the least common was visual impairment $(0.2 \%)$. Only $1.7 \%$ discontinued the treatment citing adverse drug reactions and $10.5 \%$ required permanent discontinuation of the offending drug. Conclusion: Treatment of MDR-TB is challenging mainly due to the long duration of treatment and the potential adverse reactions of the drugs used. These reactions are frequent but majority of them can be successfully managed without treatment interruption. Training the peripheral health centre workers to identify and refer the patients with adverse reaction bears a major impact on treatment outcome.
\end{abstract}

\section{Keywords}

MDR-TB, ADR, Adherence, Outcome

How to cite this paper: Akshata, J.S., Chakrabarthy, A., Swapna, R., Buggi, S. and Somashekar, M. (2015) Adverse Drug Reactions in Management of Multi Drug Resistant Tuberculosis, in Tertiary Chest Institute. Journal of Tuberculosis Research, 3, 27-33. http://dx.doi.org/10.4236/itr.2015.32004 


\section{Introduction}

Multidrug resistant tuberculosis (MDR-TB), defined as resistance to at least rifampicin and isoniazid, is a growing concern throughout the world. As per recent global tuberculosis report of WHOm the incidence of MDR-TB is $3.5 \%$ among new cases and $20.5 \%$ among previously treated for tuberculosis cases [1]. India along with China \& Russian Federation contributes to about half the load of MDR-TB cases [1].

Programmatic management of drug resistant tuberculosis (PMDT) services in India were initiated from August-2007. A standardised Cat IV regimen has been implemented by the Revised National Tuberculosis Control Programme (RNTCP) [2]. The duration of MDR-TB treatment is 24 - 27 months and multiple drugs are used in the regimen which has a great potential for adverse drug reactions. Adverse drug reactions associated with the second line anti-tuberculosis drugs used has a severe impact on adherence [3] [4] to treatment. As adherence to treatment is the key to successful outcome, identification and early management of adverse drug reactions plays a major role in MDR-TB management. Several studies have contributed to the knowledge of these adverse reactions [5]-[7]. In this study we report the occurrence of adverse drug reactions encountered during MDR-TB treatment.

Material \& Methods: Retrospective cohort study was done in SDSTRC \& Rajiv Gandhi institute of Chest Diseases, a tertiary chest institute.

Inclusion criteria: Sputum culture confirmed MDR-TB patients who received at least 3 months of MDR-TB treatment during August 2011 to December 2014 were included in this analysis.

All confirmed cases were admitted for pre-treatment evaluation as per National guidelines. Baseline investigation included clinical examination, chest $\mathrm{X}$-ray, complete haemogram, liver function test, blood urea, serum creatinine, blood sugar-fasting, post prandial, HIV (ELISA), thyroid profile, urine pregnancy test for women of child bearing age group.

Patients were initiated on standardised Cat IV regimen which includes kanamycin, levofloxacin, ethionamide, pyrizanamide, ethambutol, cyclosereinefor 6 - 9 months and levofloxacin, ethionamide, ethambutol, cyclosereine for 18 months. Para-Aminosalicylic acid was used as a reserve/substitute drug .Dosage of the drugs was as per weight bands recommended. After 10 - 15 days of initiation of treatment, patients were discharged from hospital and continued treatment from pre-identified peripheral units. As per national guidelines, patients were clinically monitored regularly. Serum creatinine was repeated every month for first 3 months and every 3 months thereafter in patients receiving kanamycin. Symptomatic patients were evaluated by chest physicians and appropriate laboratory investigations were done on advice. Specialist consultation (psychiatric, audiometric, ophthalmic, dermatologist) was taken whenever indicated.

Retrospective data was collected by reviewing patient case files, treatment cards, registers maintained at DOTS-Plus site and reports from peripheral units submitted on line. For adverse drug reactions defined by laboratory values, at least one documented abnormal value was considered. For those not defined by laboratory values, event was considered if the chest physician/pharmacovigilence team documented the reaction in the patient case file according to his/her clinical criteria (Table 1).

Management of these adverse reactions symptomatically with/without modification of the MDR-TB regimen was noted if documented in the case file/treatment card.

Exclusion criteria:

1) Patients who received less than 3 months' treatment at the time of this study.

2) Patients with abnormal laboratory value at baseline evaluation were excluded while analysing for that particular adverse effect.

The study was approved by the institutional DOTS plus and ethical committee.

\section{Results}

607out of 675 patients had received at least 3 months' treatment at the time of this study and only those were included in the study.

Majority of the cohort experienced adverse drug reactions. Many patients experienced more than one adverse reaction. Adverse drug reaction ranged from minor (gastritis, rash, nausea, diarrhoea, arthralgia) to major life threatening events (renal failure, hepatotoxicity). Table 2 shows the clinical and demographic data of 607 patients in study.

The most common of adverse drug reactions was related to gastrointestinal system. 435 (71.7\%) patients of 607 complained of mild gastritis, nausea, vomiting. Other five commonly occurring adverse reactions were ar- 
Table 1. Definition of adverse effects: [5] [6].

\begin{tabular}{cl}
\hline Side Effects & Nausea, vomiting or pain abdomen \\
\hline Gastro-intestinal & $\begin{array}{l}\text { Documented by physician } \\
\text { Joint pains with or without arthritis }\end{array}$ \\
Diarrhoea arthralgia & Elevation of Serum creatinine levels above the normal values ${ }^{*}$ \\
Nephrotoxicity & 1) Hearing loss confirmed by physical examination or audiometry \\
Oto-vestibular toxicity & 2) Vestibular side effects like dizziness/vertigo/tinnitus. \\
& Any drug reaction felt to be related to anti-tuberulous medication, as documented by TB \\
physician/dermatologist & Symptoms \& findings consistent with neuropathy eg: numbness, tingling or burning in \\
Dermatologic & trunks or extremities, diagnosed by physician or nerve conduction studies \\
Peripheral neuropathy & Seizure activities witnessed or unwitnessed \\
Seizure & Serum TSH levels $>10$ micro IU/ml \\
Hypothyroidism & Elevation of transaminases thrice the upper normal limit or/and bilirubin twice the upper \\
Hepatotoxicity & normal limit \\
& Visual changes suggestive of optic neuritis or loss of color vision confirmed by an \\
Ocular & ophthalmologist \\
& Presence of one or more of the following \\
Depression, adjustment disorders, anxiety/psychosis suicidal tendencies as diagnosed by \\
psychiatrist
\end{tabular}

*Normal range: AST (0 - $45 \mathrm{U} / \mathrm{L})$; ALT (0 - $45 \mathrm{U} / \mathrm{L})$; bilirubin (0.1 - $1 \mathrm{mg} / \mathrm{dl})$; creatinine (0.6 - $1.2 \mathrm{mg} / \mathrm{dl})$.

Table 2. Clinical and demographic data of MDR-TB patients.

\begin{tabular}{ccc}
\hline & Sex Ratio & \\
\hline Male & & 402 \\
& Female & 205 \\
\hline & Age & \\
\hline$<30$ yrs & & 231 \\
$>30$ yrs & Weight & \\
\hline & & 876 \\
\hline$<25$ kgs & 599 \\
\hline$>25$ kgs & Disease & 159 \\
\hline New (cat I-failure) & & 448 \\
\hline Re-Treatment (Cat II-failure) & Demography & 430 \\
\hline Urban & & 177 \\
\hline Rural & & \\
\hline
\end{tabular}

thralgia (14\%), depression (13\%), diarrhoea (8.6\%), peripheral neuropathy (5.8\%) and skin rash (4.3\%). The least occurring were nephrotoxicity $(0.5 \%)$ \& ocular $(0.2 \%)$ (Table 3). Although majority of the patients had an adverse drug reaction, only $1.7 \%$ stopped the treatment. Only 64 (10.5\%) patients required permanent discontinuation of the offending drug from the regimen due to adverse drug reaction.

Most of the adverse drug reactions were managed on outpatient basis with symptomatic treatment. Figure 1 shows the flow of treatment in the study population. Offending drugs were either reduced in dose or temporarily suspended. Re-introduction of the drug was generally attempted after improvement of symptoms.

\section{Discussion}

Adverse drug reactions during MDR-TB treatment reported by Indian studies varied from $57.14 \%$ to $94.3 \%$ [3] 


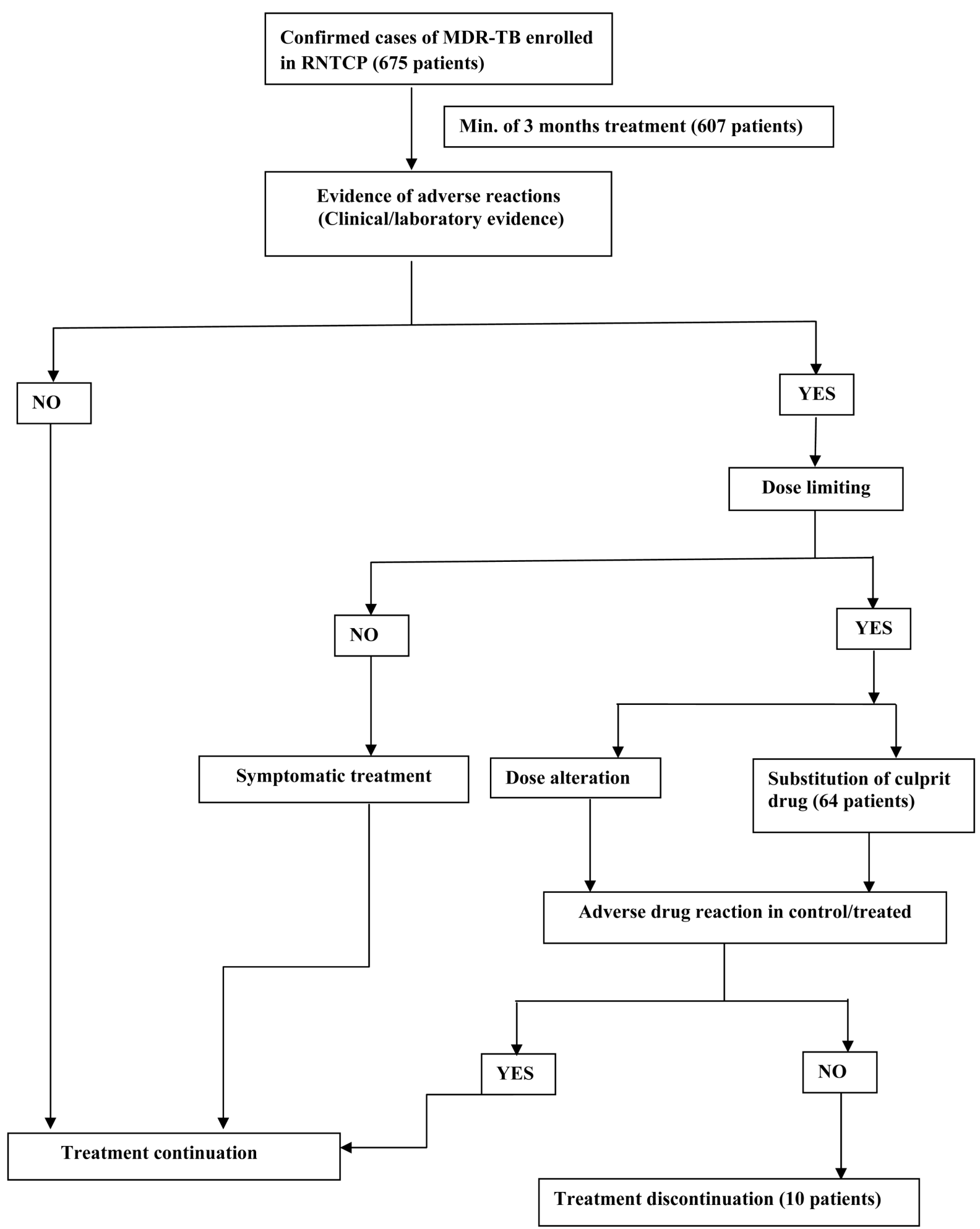

Figure 1. Treatment pattern in patients of MDR-TB.

[4]. In our cohort of 607 patients treated with standardised Cat IV regimen, majority experienced one or more adverse drug reactions.73.3\% of 244 patients, consecutively enrolled into the DOTS-Plus program in Tomsk, Russia reported adverse drug reactions. Though the study involved individualised regimen, it generally included 
Table 3. Frequency of adverse drug reactions.

\begin{tabular}{ccc}
\hline Adverse reactions & No. & $\%$ \\
\hline Gastrointestinal (nausea, vomiting, pain abdomen) & 435 & 71.1 \\
Nephrotoxicity & 3 & 0.5 \\
Hepatotoxicity & 7 & 1.2 \\
Hypothyroidism & 19 & 3.1 \\
Depression & 78 & 13 \\
Psychosis & 10 & 2.6 \\
Seizures & 15 & 3 \\
Oto-vestibular toxicity & 18 & 14 \\
Arthralgia & 85 & 4.3 \\
dermatologic & 26 & 5.8 \\
Neuropathy & 35 & 0.2 \\
Opthalmic & 1 & 8.6 \\
\hline
\end{tabular}

a parenteral aminoglycosides, PAS, thioamides \& cycloserine [5].

Although majority of our cohort explained adverse drug reactions, only $1.7 \%$ discontinued the treatment. These findings are similar to the experience reported from five DOTS plus pilot projects, where only $2.1 \%$ stopped the treatment due to adverse drug reactions [7]. Other studies done in Tomsk, Russia \&Lima, Peru reported that none of the patient required discontinuation of the entire treatment due to adverse reaction alone [5] [6].

But $10.5 \%$ of the patients required permanent discontinuation of the offending drug. This was comparable to study from Lima, Peru which reported $11.7 \%$ required discontinuation of offending drug [6]. But this was significantly less compared to other studies reporting $28.7 \%$ and 30\% [5] [7] (Table 4).

Gastrointestinal adverse reactions were the commonest (71.7\%) in our study which was similar to other studies reported in India \& outside [3]-[7] (Table 5).

Depression was noted in 78 (13\%) patients of our cohort which ranged from mild to severe suicidal tendencies. Other studies reported depression between 6.2\% - 18.3\% [5]-[7]. A study from Egypt showed that 26.5\% MDR-TB patients had depression [8]. Cycloserine being the culprit drug was discontinued in few severe cases and in majority it was continued along with anti-depressant therapy. Socio-economic problems, chronicity of the disease may have added to depression in many cases.

Hypothyroidism was reported less frequently (3.1\%) in our study. In comparison few studies reported hypothyroidism ranging from 10\% - 39.5\% [5] [6] [8]. In our study, as per the guidelines TSH was done at baseline and was repeated on clinical suspicion. The subtle symptoms of hypothyroidism may be either missed by the clinicians or attributed to unpleasant effect of drugs, so may be under-diagnosed. Also the other studies used combination of both PAS and thioamides as frequent drugs in the regimen as compared to our study where ethionamide was the only drug. PAS was used as a substitute drug, in our study for only the life threatening adverse drug reaction or an initial resistance to any second line drugs [5] [6] [8].

Ototoxicity, hepatotoxicity and nephrotoxicity were observed less frequently in our study. Nephrotoxicity was documented in only $0.5 \%$, in spite of regular monitoring of serum creatinine levels every month during first 3 months and every 3 months thereafter till kanamycin was administered. Liver function tests were also frequently repeated in most of the patients who presented with GI disturbance/loss of appetite. Frequent monitoring for these potential adverse reactions, not only clinically but also by well-defined laboratory criteria, may be the reason for appropriate management and thus minimizing the life threatening impact of these.

Our study has certain limitations. It being a retrospective study, adverse drug reactions may be under-reported/over-reported, which is there may be documentation errors. Adverse reactions which lacked definite laboratory criteria were relied on physicians' documentation leading to reporting bias. Few adverse drug reactions 
Table 4. Rate of complete cessation and discontinuation of offending drug.

\begin{tabular}{ccccc}
\hline & Tomsk, Russia [5] & DOTS Plus pilot projects [7] & Lima, Peru [6] & Present \\
\hline $\begin{array}{c}\text { Regimen type } \\
\begin{array}{c}\text { Permanent discontinuation of } \\
\text { offending drug }\end{array}\end{array}$ & Individualised & Individualised & Individualised & Standardised \\
Complete stop of treatment & $28.70 \%$ & $30 \%$ & $11.70 \%$ & $10.50 \%$ \\
\hline
\end{tabular}

Table 5. Adverse drug reactions in different studies.

\begin{tabular}{|c|c|c|c|c|}
\hline & Tomsk, Russia [5] & DOTS Plus pilot projects [7] & Lima, Peru [6] & Present Study \\
\hline Study type & Retrospective & Retrospective & Retrospective & Retrospective \\
\hline No of cohort & 244 & 818 & 60 & 607 \\
\hline \multicolumn{5}{|l|}{ Adverse reactions } \\
\hline & $\%$ & $\%$ & $\%$ & $\%$ \\
\hline $\begin{array}{c}\text { Gastrointestinal } \\
\text { (nausea, vomiting, pain abdomen) }\end{array}$ & 75.4 & 61.2 & 100 & 71.1 \\
\hline Nephrotoxicity & 9.8 & 1.2 & 3.3 & 0.5 \\
\hline Hepatotoxicity & 16.8 & 2.2 & 1.7 & 1.2 \\
\hline Hypothyroidism & 17.2 & 3.5 & 10 & 3.1 \\
\hline Depression & 8.6 & 6.2 & 18.3 & 13 \\
\hline Psychosis & 11.9 & 3.4 & 10 & 1.6 \\
\hline Seizures & 11.5 & 4 & 8.3 & 2.5 \\
\hline Oto-vestibular toxicity & 15.6 & 12 & 6.7 & 3 \\
\hline Arthralgia & 47.1 & 16.4 & 6.7 & 14 \\
\hline Dermatologic & 16 & 4.6 & 43.3 & 4.3 \\
\hline Neuropathy & 4.1 & 7.9 & 20 & 5.8 \\
\hline Opthalmic & - & 4.4 & - & 0.2 \\
\hline Diarrhoea & 46.3 & 21.1 & _ & 8.6 \\
\hline
\end{tabular}

may have been missed by the treating physician. Regular clinical monitoring of the patients as per programmatic guidelines was difficult in some non co-operative, non adherent cases. Some laboratory parameters like liver function tests and TSH were not repeated at regular intervals. They were done as and when required if clinician suspected symptoms.

\section{Conclusion}

MDR-TB treatment is a major challenge given the chronic nature of disease, long duration of treatment and multiple drugs used in the regimen. The wide spectrum of potential adverse drug reactions further escalates this challenge. As we could see in our study, though adverse drug reactions were frequently reported, majority continued the treatment with either supportive treatment or discontinuation of offending drugs. Non adherence to treatment due to any reason can negatively impact the treatment outcome. Prompt identification and management of adverse drug reactions holds the key to successful outcome. Under programmatic conditions, training of primary health care workers to detect adverse drug reactions, development of management protocols feasible at peripheral centres and prompt referral to higher centres if required, can have a major impact on treating the adverse reactions and hence the management of MDR-TB.

\section{References}

[1] WHO (2014) Global Tuberculosis Report 2014—Drug Resistant TB: Surveillance and Response. 
[2] Central TB Division (CTD), Directorate General of Health Services, Ministry of Health and Family Welfare, Government of India (2006) DOTS-Plus Guidelines. CTD, New Delhi.

[3] KapadiaVishakha, K. and Tripathi Sanjay, B. (2013) Analysis of 63 Patients of MDR TB on DOTS Plus regimen: An LG Hospital, TB Unit, Ahmedabad Experience. Gujarat Medical Journal, 68, 52-57.

[4] Joseph, P., Rao Desai, V.B., Mohan, N.S., Fredrick, J.S., Ramachandran, R., Raman, B., et al. (2011) Outcome of Standardized Treatment for Patients with MDR-TB from Tamil Nadu, India. Indian Journal of Medical Research, 133, 529-534.

[5] Shin, S.S., Pasechnikov, A.D., Gelmanova, I.Y., Peremitin, G.G., Strelis, A.K., Mishustin, S., et al. (2007) Adverse Reactions among Patients Being Treated for MDR-TB in Tomsk, Russia. The International Journal of Tuberculosis and Lung Disease, 11, 1314-1320.

[6] Furin, J.J., Mitnick, C.D., Shin, S.S., Bayona, J., Becerra, M.C., Singler, J.M., et al. (2001) Occurrence of Serious Adverse Effects in Patients Receiving Community-Based Therapy for Multidrug-Resistant Tuberculosis. The International Journal of Tuberculosis and Lung Disease, 5, 648-655.

[7] Nathanson, E., Gupta, R., Huamani, P., Leimane, V., Pasechnikov, A.D., Tupasi, T.E., et al. (2004) Adverse Events in the Treatment of Multidrug-Resistant Tuberculosis: Results from the DOTS-Plus Initiative. The International Journal of Tuberculosis and Lung Disease, 8, 1382-1384.

[8] Elmahallawy, I.I., Bakr, R.M., Mabrouka, A.A. and Omar, R.M. (2012) Treatment Outcomes among Patients with Multi-Drug Resistant Tuberculosis in Abbassia Chest Hospital from July 2006 to June 2010. Egyptian Journal of Chest Diseases and Tuberculosis, 61, 337-342. http://dx.doi.org/10.1016/j.ejcdt.2012.08.018 